\title{
A ZHLS Based Geocasting for Broadcasting Messages in VANET
}

\author{
Wang-Cheol Song and Muhammad Shoaib
}

\begin{abstract}
Geocasting is used for dissemination of messages in defined geographical locations. It is very useful in traffic information system where warning messages are openly needed to be disseminated in different areas. One major problem in VANET geocasting is that if there exist islands that are not directly connected within the geocast region, the delivery become impossible. In order to overcome this issue in this paper a new Zone based Geocasting algorithm is presented in this paper. The area is divided into different zones including roads. Routing path is defined in form of zones, instead of GPS region or nodes in order to make sure that message is delivered to all zones in the geocast region. Furthermore if there are islands in the geocast regions, they are identified by zone database. Different routing paths are set for multiple islands to make sure they got the geocast message. Our experiments prove using zone based algorithm better performance in term of delivery guarantee can be achieved.
\end{abstract}

Index Terms-VANET, geocasting, ZHLS, delivery guarantee, island.

\section{INTRODUCTION}

In geocasting, a message is required to be disseminated in a certain area extending beyond the sender's transmission range [1], [2]. When the sender is located outside the geocast region the message is transmitted though the intermediary vehicular nodes. These messages need to be disseminated to all the nodes present in the geocast region particularly when a safety-related or warning message is geocasted. This approach is beneficial in VANETs, because most warnings and information messages are subjected to relate with a certain area rather than individual vehicles. However it suffers from many limitations. Among these limitations; one limitation that current geocasting protocols [1]-[4] presented in the domain of VANET does not address the guarantee delivery. If the geocast region is composite of islands, i.e. some parts of the region are not in transmission range each other, messages cannot be deliver to that parts that can be named islands. Fig. 1 presents an example of such scenario where geocast region is divided into two island $\operatorname{Road} A$ is disconnected from Road $B$ and Road $C$. The message in this case needs to be delivered to nodes present at Road $A$ and Road $B$ separately in order to ensure the deliver to all nodes in

Manuscript received March 20, 2014; revised June 10, 2014. This research was supported by Basic Science Research Program through the National Research Foundation of Korea (NRF) funded by the Ministry of Education, Science, and Technology (2011-0012329).

Wang-Cheol Song is with the Department of Computer Engineering, Jeju National University, South Korea (email: philo@jejunu.ac.kr).

Muhammad Shoaib is with Department of Research \& Development, Institute of Space Technology, Pakistan (email: muhammad.shoaib@live.com). the geocast region.

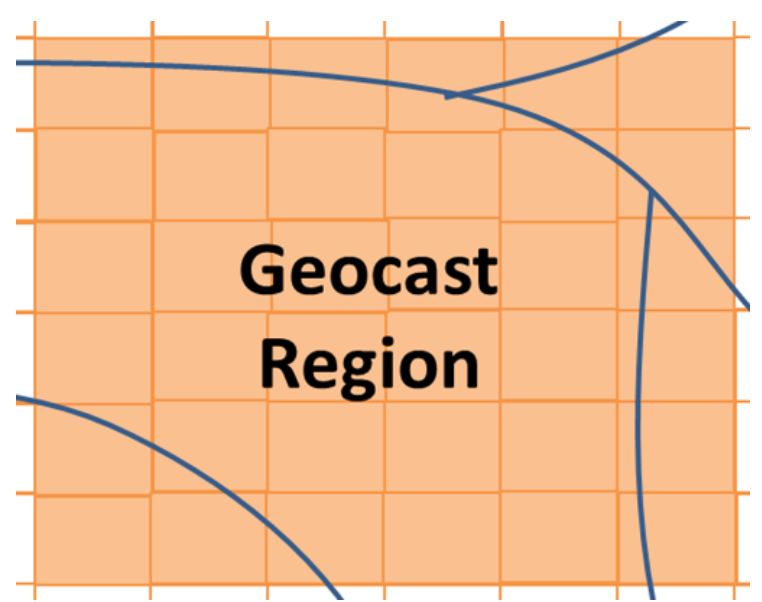

Fig. 1. An example of island based geocast region

In order to overcome this issue in this paper we propose a geocasting method based on zone-based hierarchical LSR protocol (ZHLS) [5] for geocasting messages in VANET environment. A node in ZHLS uses the location information only to know its position, and exchange information about the connectivity of the networks to know the network topology (similar to how existing routing algorithms for wired networks work), so that a node is not concerned with encountering a dead end while searching for a path to a destination. This addresses a problem typically found in location-aware or geocast routing algorithms. Therefore, we suggest using ZHLS to guarantee delivery of geocast packets to all nodes of each island in the geocast region by using topology information provided by ZHLS. Another rationale for using ZHLS is its support for high mobility. We take the benefit of its two tire topology i.e. zone level topology and node level topology. The end-to-end path in ZHLS is first defined in term of zone level topology that does not change as frequently as the node level topology change. When a packet reaches a destination zone, node level topology is used to route the packet. As zones topology in VANET should be based on road's information ZHLS is more suitable for VANET instead of MANET despite of the factor that it was originally proposed for MANET.

Our system is for a mechanism for a remote node to send geocast packet to a specific region. As shown in Fig. 2, a node in the geocast point can send packets to a geocast region. The packets arrived at a point in the geocast region cannot be delivered to all nodes in the region because some parts of the zones are not connected each other within the geocast region. In this case the node in the geocast point should first recognize the islands in the region and separately send packets for every island. 


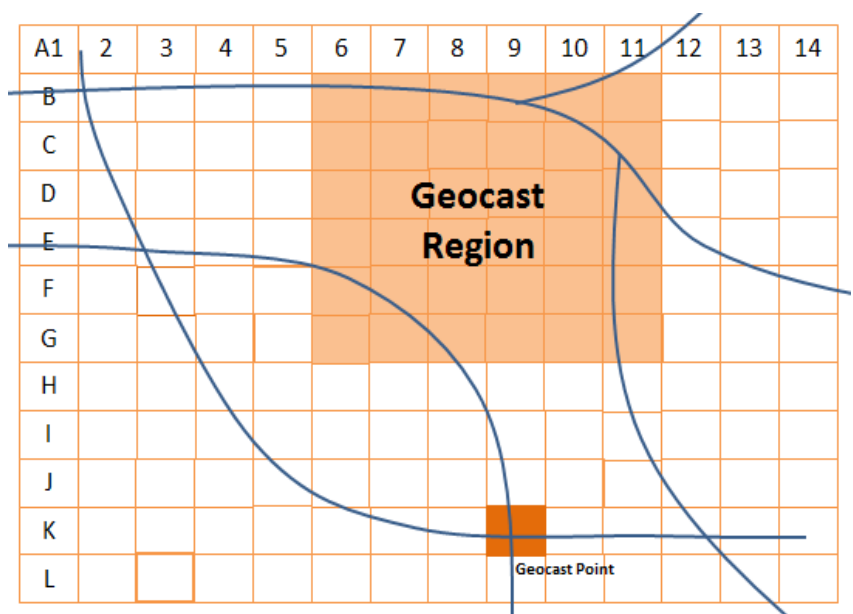

Fig. 2. Sample Zone map for geocasting.

The rest of paper is organized as follows; in Section II where we have discussed our algorithm based on ZHLS. Section III we discuss our experiments and results followed by Section IV where paper is concluded.

\section{Proposed Algorithm For DeliVery GuARANTEE}

This algorithm is for a case that a node in a remote site wants to geocast packets to a region with guaranteed delivery based on ZHLS. The Zone LSP shows the connectivity of zones in the geocast region. When a packet is sent to a geocast region, a path to each island in the geocast region can be determined by investigating the Zone LSP. When any zone topology changes occur, it can be recognized by a newly generated Zone LSP.

As each node in ZHLS asynchronously floods its Zone LSP, the further the destination geocast region is from the sender or the faster the nodes in VANETs move, the less accurately the sender's Zone LSP may be reflective of the topology of the remote geocast region. In order to get the most accurate topology information for the geocast region, it can be suggested that the sender of the geocast packets gets the Zone LSP from gateway nodes of some zones in the region. Once Zone LSPs for the destination region is obtained, the steps for sending packets are as follows:

1) The sender gets Zone LSPs from gateway nodes of the zones in the geocast region, and checks whether all of Zone LSPs are the same. If this is not the case, then each row of the resultant Zone LSP is filled with the corresponding row from Zone LSP of each zone

2) The sender investigates the Zone LSPs to discover the Zone Level topology in the geocast region. The algorithm for searching islands is described in the section 3.

3) The sender determines a proper zone to receive the geocast packets for each island. When a zone to receive the packets is determined, the packet can be routed to a proper gateway node of the zone by ZHLS routing algorithm.

4) The sender unicasts the geocast packets to a gateway node of each zone associated with an island. A packet may consist of the following elements. (Fig. 3).

- Sender ID is Node ID

- Receiver Location is represented as geographical location points such as X1Y1, X2Y2, X3Y3, X4Y4

- An LSP is composite of nodes and zones composition.

- More than one LSPs are inserted from the sender based on his LSP Database if multiple locations end points or there

- Active LSP means which LSP is being used for current path

- Message Validity is the Date and Time the message a validate to discard the outdated packets if needed

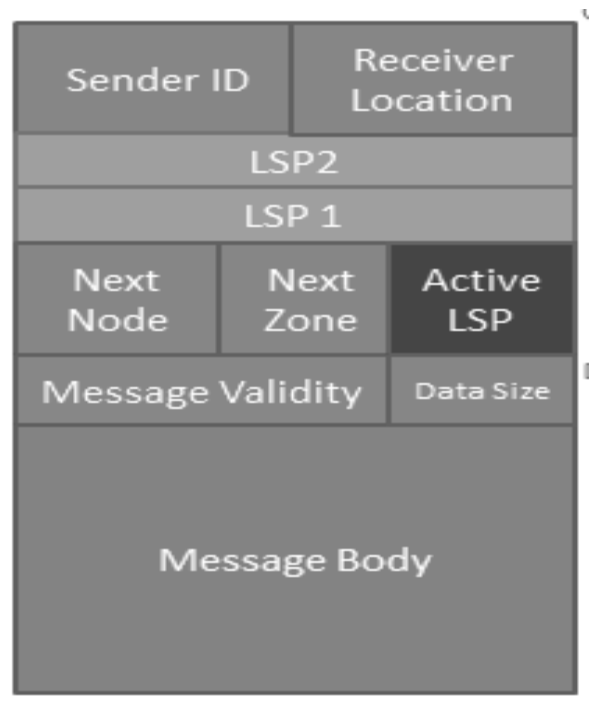

Fig. 3. A proposed message packet format.

5) The packets received by the gateway nodes are geocasted inside the geocast region.

In order to perform geocasting with the proposed procedure, it is assumed that the sender sends the geocasting packets with zone IDs for indication about the geocasting area in the header, and every node has the capability to geocast packets within indicated area.

In ZHLS algorithm, the gateway nodes do not broadcast a Zone LSP if its value is the same as the old Zone LSP. So, it is possible to make the gateway node send the newly-changed Zone LSP to the sender. Through this, topology change in the geocast region can be recognized by the sender so that geocast packets can be properly delivered according to the changed topology of islands. However, the zone topology is usually very stable, we expect that at least one time geocast can be delivered to nodes in the geocast region after Zone LSPs are gotten

\section{IDENTIFYING THE ISLANDS}

It is assumed that the sender sequentially numbers all zones in the geocast region and zone numbers in received Zone LSPs are converted the sequential numbers for the sake of convenience when the sender receives Zone LSPs for geocast region.

The variable island[number of islands][queue length] stores connectivity between zones. The variable $i \_z o n e$ [number of islands] is for identifying which zones are included in a specific island bit-by-bit.

1) Start identification of the islands in the geocast region at the first row of Zone LSP with the number of island $=0$. 
2) If zone $i$ in the first row has some numbers such $j, k$ and $l$ as the connected zone number, put the number $i, j, k, l$ to the queue island[0][] and set the $i$ th, $j$ th, $k$ th and $l$ th bits of $i \_z o n e[0]$. Then, island[0][0] $=i$, island $[0][1]=j$, island $[0][2]=k$, and island $[0][3])=l$.

3) Go to $j$ th row in Zone LSP and get the numbers. Compare the numbers with each bit of the corresponding order in i_zone [0], and ignore the numbers that have been already included in $i_{-} z o n e[0]$. Remaining numbers should be put to the queue - island[0][], and set a bit in the order of each number of $i \_z o n e[0]$ as like step 2.

4) Repeat step 3 until all numbers in the queue - island[0][] are processed. Then, which zones belong to island[0][] can be easily identified by using bits of i_zone[0]. Then, island[0][] has all zone numbers included in island $\mathbf{0}$, and $i_{-} z o n e[0]$ provide a way to know at a glance which zones are included in island $\mathbf{0 .}$

5) If there are any zones not included in island $\mathbf{0}$ among zones in the geocast region, repeat step from 1 to 4 with island[1][] and $i_{\text {_zone }}[1]$ for island $\mathbf{1}$.

6) Go further steps as like step 5 until all zones in geocast region belongs to islands.

\section{EXPERIMENTS AND RESULTS}

We have simulated our algorithm for an environment depicted in Fig. 4 using NS2 simulator. A real map of Seoul, Korea has been used for the experiments. Experiments have been conducted for different velocities of vehicles that vary as 20, 40, 60, 80 and 100 with the different density for each velocity. Table I shows the relationship of vehicle velocity and density used in our experiments. Geographical locations are divided into different zones where zone information database is inserted into each vehicular node. One Zone is covered by $1 \mathrm{KM}$ that is equal to communication range of IEEE 802.11p. Thus in our normal simulation there are no multiple hops in one zone because all nodes in a zone remain in each other's communication range.

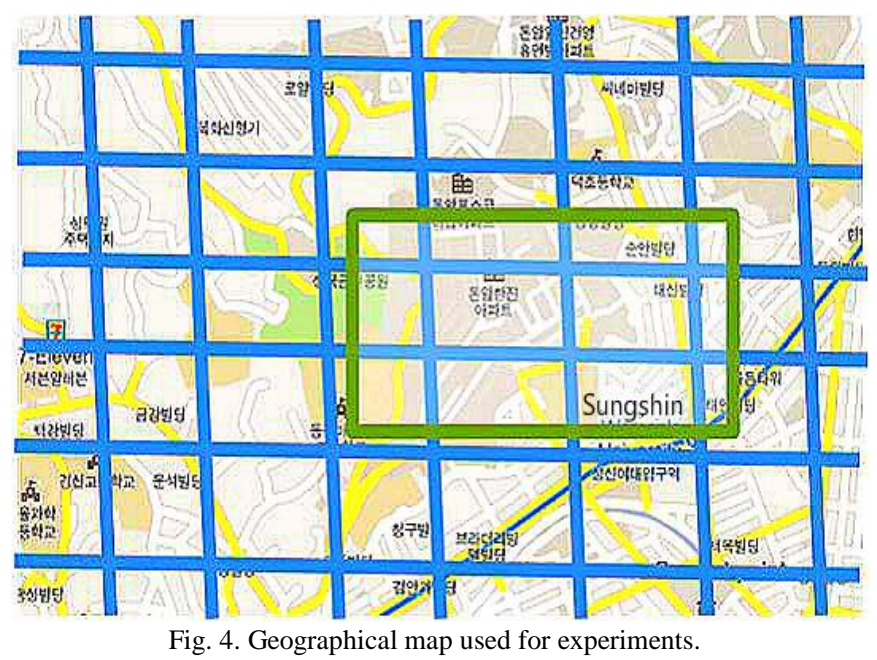

The experiments were conducted to measure the performance of ZHLS for Geocasting in region. Experiments were conducted 10 times and presented results are the average of results obtained in all turns.

TABLE I: CLASSIFICATION OF VEHICULAR NODES
\begin{tabular}{|l|l|l|}
\hline Nodes & Density & Velocity \\
\hline 100 & 2 per zone & 100 \\
\hline 200 & 4 per zone & 80 \\
\hline 300 & 6 per zone & 60 \\
\hline 400 & 8 per zone & 40 \\
\hline 500 & 10 per zone & 20 \\
\hline
\end{tabular}

Fig. 5 shows the results for path stability. Path stability was computed with the ratio of path changing on zone level. There was only one observed case in ZHLS when zone level path changed and that specific case came when all nodes left a specific zone. Number of nodes was a major factor for the path stability. The more number of nodes build the more stable path. Therefore we can say ZHLS based geocasting is more useful in urban areas instead of rural area.

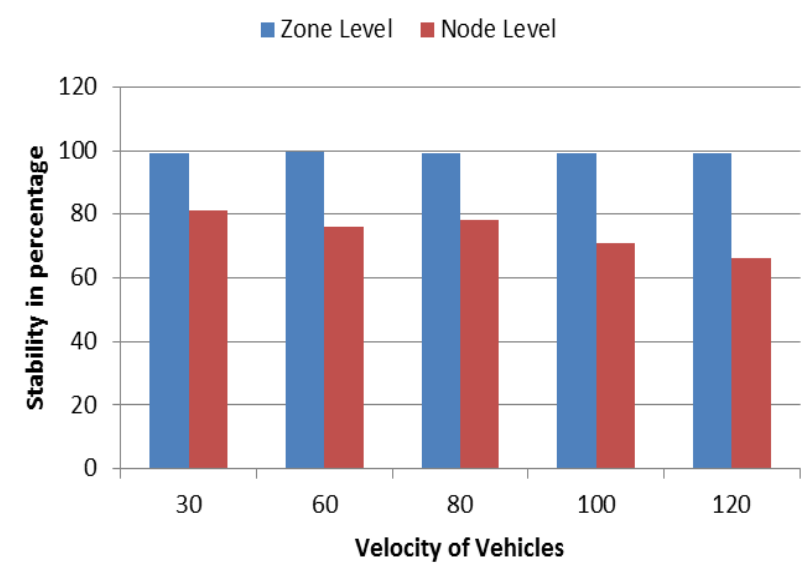

Fig. 5. Stability of path.

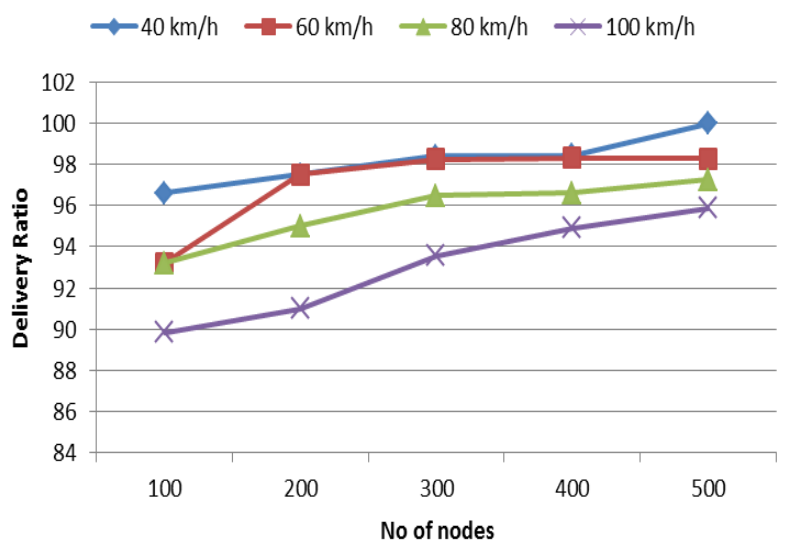

Fig. 6a. Overall delivery ratios with respect of nodes.

The experimental parameters used to test the algorithm are for performance measurement. We evaluate our algorithm with the percentage of delivery within geocast region i.e. how many nodes within the geocast region receive the geocast packet. For this purpose total of 10 geocast packets were sent for different geocast region. Each region designed is based on islands so that more than one LSP can be inserted in the packet. Fig. 6 shows the delivery ratios as the results which is no less than $86 \%$. 


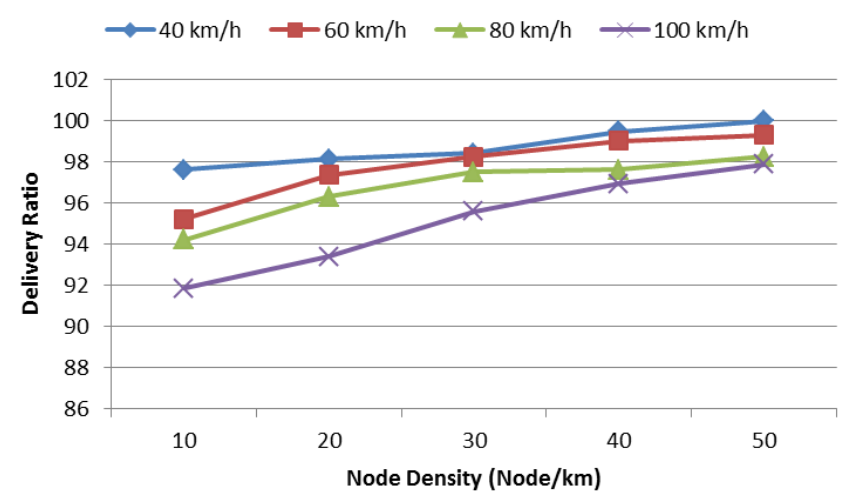

Fig. 6b. Overall delivery ration with respect to node density.

The delivery ratio depended on the density of the nodes and that is natural. Our experiments show that the more the number of nodes present in the nodes, the more delivery rate is observed. The reason for this is simple as the less density means the low connectivity. If a node cannot find the next node needed for moving in the packet in the next zone in a certain amount of time, it drops the packet. Therefore the packets drop ratio is exactly inversely proportional to the delivery ratio.

\section{CONCLUSIONS}

We have presented a geocasting protocol for vehicular ad hoc networks. The main idea is to use the ZHLS approach for geocasting warning messages in a defined geographical area. We have taken a benefit of two layer topology of ZHLS i.e. node level topology and zone level topology in a highly dynamic environment where nods change their location very frequently. As the message is routed based on the zone topology, changes in the node level topology doesn't really create much effect in performance. Also zone based approach is quicker and more stable then flooding based approaches because of stability of its zone level topology. Our experiments shows that zone level topology does not change as frequently as the node level topology change therefore a low drop rate and high delivery ratio was observed.

\section{REFERENCES}

[1] F. Li and Y. Wang, "Routing in vehicular ad hoc networks: A survey," IEEE Vehicular Technology Magazine, vol. 2, no. 2, pp. 12 -22, 2007.

[2] W. Wang, F. Xie, and M. Chatterjee, "An integrated study on mobility models and scalable routing protocols in VANETs," in Proc. Mobile Netw. Veh. Environ., 2007, pp. 97-102.

[3] M. Al-Rabayah and R. Malaney, "A new hybrid location-based ad hoc routing protocol," in Proc. IEEE GlobeCom, 2010, pp. 1-6.

[4] V. Namboodiri and L. Gao, "Prediction-based routing for vehicular Ad hoc networks," IEEE Transactions on Vehicular Technology, vol. 56, no.4, pp. 2332-2345, July 2007.

[5] M. J.Ng and I. T. Lu, "A peer-to-peer zone-based two-level link state routing for mobile ad hoc networks," IEEE Journal on Selected Areas in Communications, vol. 17, no. 8, pp. 1415-1425, Aug 1999.

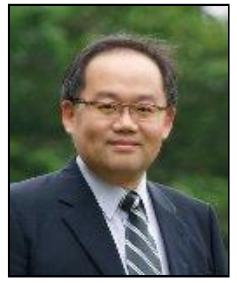

Wang-Cheol Song received the B.S. degree in food engineering and electronics from Yonsei University, Seoul, Korea in 1986 and 1989, respectively. And he received his M.S. and $\mathrm{PhD}$ in electronics from Yonsei University, Seoul, Korea, in 1991 and 1995, respectively. Since March 1996, he has been a professor of Department of Computer Engineering, Jeju National University, Korea. His research interests include VANETs and MANETs, future internet, network security, and network management.

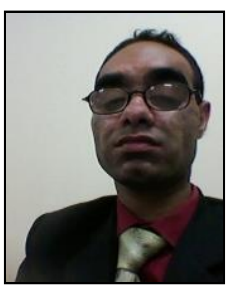

Muhammad Shoaib received the bachelor of science in computer science from National University of Computer and Emerging Sciences, Islamabad, Pakistan in 2010. And he received his master of engineering in computer engineering from Jeju National University, Jeju, Korea in 2013. He is working for Department of Research \& Development, Institute of Space Technology, Pakistan. His research interests include data mining, complex networks, information retrieval, semantic web, data storage and querying. 